\title{
Ergonomic Evaluation and Customized Design of Kitchen
}

\author{
Amit Bhatia, Sandeep Singla
}

\begin{abstract}
Ergonomics is the science of planning the environment for comfortable working. Ergonomic plays an important role in designing a kitchen area free from fatigue, decreasing the unnecessary movements and excessive expenditure of workers energy and time. A poorly planned kitchen construction affects work efficiency requiring more effort and more time while working on the poorly designed kitchen counters. Kitchens vary from area to area which may or may not be designed on the basis of ergonomics. However, some women working in the kitchen experience discomfort or injury when working in the kitchen. A standard-design of a kitchen was considered as reference to have the optimal dimensions of the various components of the kitchen. For this, first a survey questionnaire was prepared to know about the problems encountered by the women working in kitchen. Anthropometric data of 30 participants from different cities was collected. The kitchen and its counters heights were designed on the basis of the anthropometric data of the same participants. Rapid upper limb assessment (RULA) and Rapid entire body assessment (REBA) Employee assessment worksheet was used for the analysis of postures of kitchen workers while working in the Standard-designed kitchen. Images of various postures of all the women volunteers were captured while working in the kitchen. After analysis of which, it was concluded that the participants were working exceeding the safe limit. Again the participants were asked to work in the kitchen designed on the basis of anthropometric data. The same procedure was followed and the results were evaluated for both standard-designed kitchen and ergonomically designed kitchen. Subsequently, it was inferred that there is a lack of ergonomics awareness among the kitchen workers and its designers. Assessment of postures using REBA and RULA shows that the majority of women are working beyond their safe limit in the standard-designed kitchen. In future, the work can be done to rationalize the kitchen dimensions that should be used in its designing.

Index Terms: Anthropometric data, Ergonomics, Kitchenette, MSD (Musculoskeletal disorders), Optimal dimensions, REBA, RULA, Standard-designed kitchen.
\end{abstract}

\section{INTRODUCTION}

Ergonomics is the study of individuals and their relationship with the environment around them. It is the practice of designing or arranging workplaces and goods with the purpose that they fit the individuals who use them. It is an approach to deal with a number of body related problems such as work-related musculoskeletal disorders. A major cause of injury for kitchen personnel besides burns, slips and falls are back strains and strains in the muscles of the upper extremity, carpal tunnel syndrome, tendonitis, and other musculoskeletal injuries. Good ergonomic design eliminates incompatibilities amongst the work and the worker and forms the optimal work environment.

Revised Manuscript Received on June 15, 2019

Amit Bhatia, Sandeep Singla, RIMT University, Punjab, India

In Ergonomics, anthropometric data (measurements of the hand, arm, elbow etc.) is applied to a product to design the shape and size of that product.

Kitchen ergonomics aims in minimizing the movements and pressure which women encounters in the kitchen while working there. Working in a kitchen is a profession requiring the use of full body movements including hand and wrist movements, bending and raising etc. The kitchen is one of the most used spaces of a house, its ergonomics are primarily important, regardless of how healthy or strong our body might be. While designing the kitchen it is the basic need to keep in mind the heights of various surfaces, distances and placement of items to make the kitchen space functional, efficient and enable effortless working in the kitchen. According to a National survey (Ministry of Statistics and Programme Implementation, 1999), in Indian, a woman spends around 3 hours 20 minutes in a day in the kitchenette, on an average, for cooking food and washing utensils. A study detected that women in India devote almost 5 to 6 hours in a day in the kitchenette [1]. This may perhaps be equal to nearly one quarter of their lifespan, which is also a reason for various health complications. Thus the working environment and area of kitchen plays a vital role in having an effect on the worker's health. If the design of the workplace is not conforming to the principles of ergonomics, then it may result in different work related Musculoskeletal Disorders (MSD). Erna Meyer (1927) addressed the matter of enhancement of work ease in houses [2]. She suggested implementing the height of kitchen furniture to be adjusted to the user's height (Fig. 1).

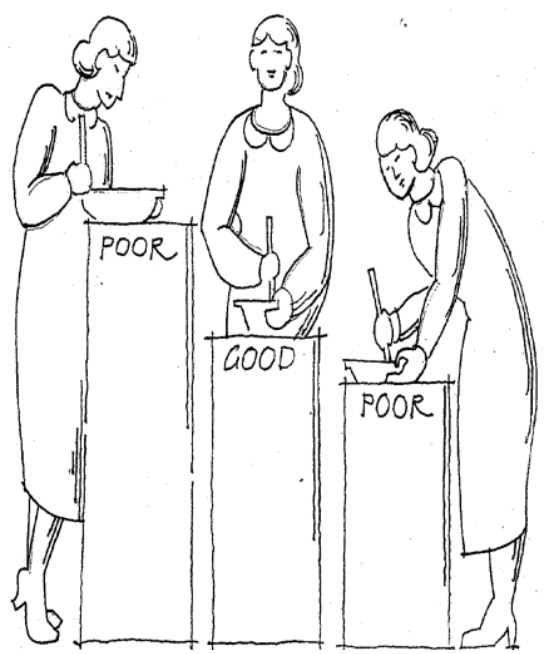

Fig. 1. Furniture in the Kitchen accustomed to the height of its users by E. Meyer (Source: Kitchen Chores Ergonomics: Research and Its Application, 2018)

Ergonomic comfort in the kitchen can be achieved through proper design of the kitchen components and layout of the kitchen. Women have to work harder in the kitchen and their

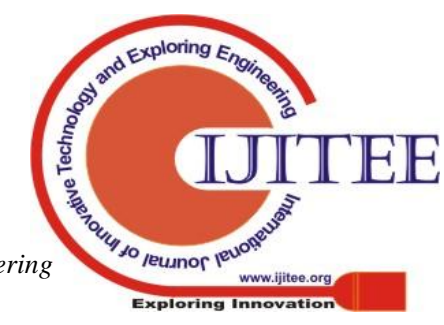


optimum efficiency cannot be attained mainly because work place is not ideally designed. Hence in order to acquire maximal efficiency in work with minimum loss to the body, it is necessary to have an ideal relationship between work, worker and workplace [3]

Work place is a significant dimension that speeds up the activity and put forth least stress on the workers. It is adequate as per the anthropometric dimensions of the worker. Females were exposed to more stress as the demands of household activities caused maximum discomfort in the faulty or inappropriate kitchen designs [4]. One of the utmost significant health problem faced by a women while working in a kitchen is pain perceived extremely at lower and upper back region. Posture and way of work had an excessive influence on the evolution of Musculoskeletal Disorders (MSD) amongst women involved in various kitchen activities [5].

Lack of information about ergonomics is seen in the industry in which work is executed. Musculoskeletal problems are there in the welding procedure where laborers are working in stooping stance and it demonstrates that they need to change the body stances [6].

Maguire et al, 2011 in their paper concluded that all the complications of reaching, stooping and stretching (Fig 2.); finesse and sight were comparatively common whereas for specific tasks, complications with ironing and cleaning were the most prevalent [7]. Some of the most common ergonomic problems are reaching window above the basin, reaching up to the topmost shelf and bending to lowermost shelf. Kitchen ergonomics is all about controlling the kneeling, bending and over-reaching. Most of the tasks in the kitchenette are done in standing position owing to this, females always encounters extreme tiredness after kitchen work particularly in the before noon time.

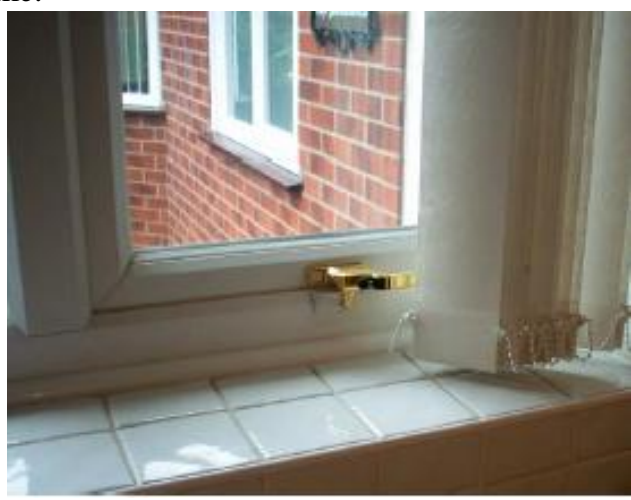

Fig 2(a)

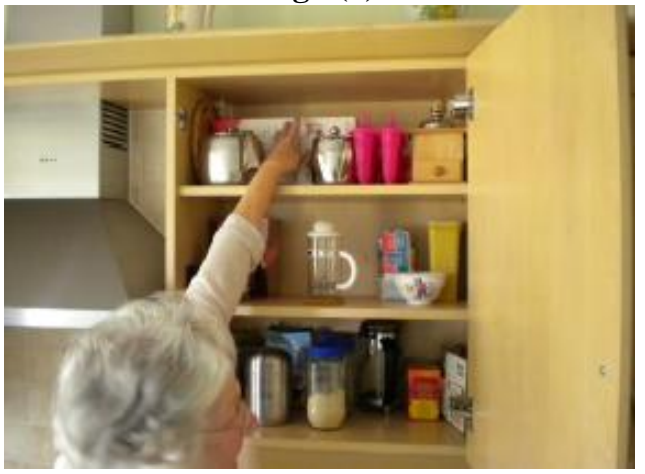

Fig 2(b)
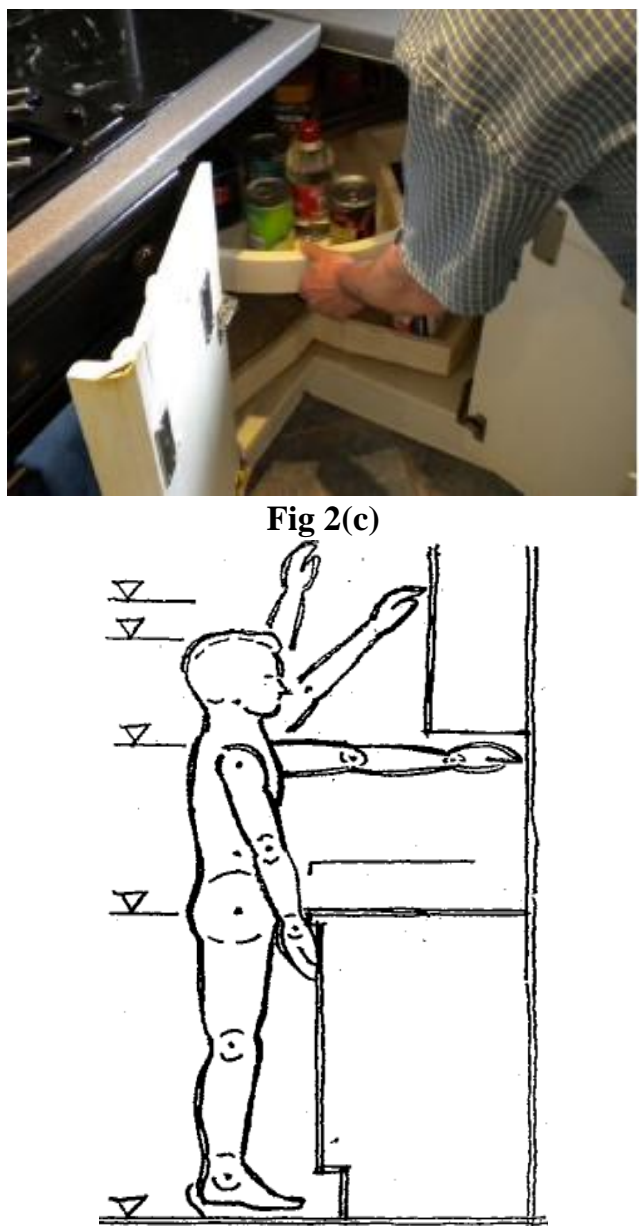

Fig 2(d)

Fig 2. Stretching and Reaching: (a) Knob at base of window makes it accessible to reach, (b) Reaching the Cupboards, (c) bending (Source: Kitchen Living in Later Life: Exploring Ergonomic Problems, Coping Strategies and Design Solutions, 2014) (d) Reaching while working on kitchen countertop. (Source: Kitchen Chores Ergonomics: Research and Its Application, 2018)

RULA (rapid upper limb assessment) is a review technique created for use in ergonomics examinations of work environments where job-related upper limb complaints are encountered [8].

Other concern is the modification of kitchen equipment on the basis of anthropometry and working capabilities of its users, in the meantime providing optimal work conditions needs preserving correct posture and minimizing muscle strains while performing various activities. The chief ergonomic risk factors consist of:

- High task repetition

- $\quad$ Forceful exertions

- Repetitive or sustained awkward postures

- Static postures

- Contact stress.

Repetitive actions like dicing and chopping vegetables, inconvenient positions like holding the head down to prepare food or bending during placing utensils, overextending like reaching for materials on boards or reaching for upper cupboard's, lifting like placing utensils and pots are the most usually done during working in the kitchenette. Postures and its preservation play a

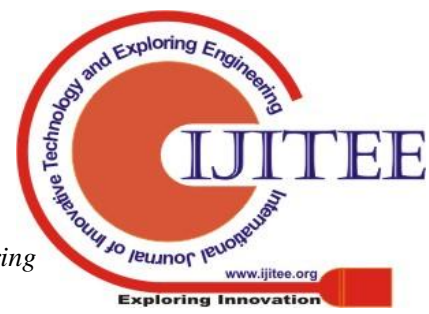


vital role in minimizing muscles related_stress.

Sultana, Sajida and Prakash, Chitra (2014) conducted a study on a sample of 1000 homemakers from Chennai city for survey with 500 using ordinary and 500 using modular kitchens [4]. To collect the information from the home makers, questionnaire method was used. The data was collected, tabulated and analyzed statistically. He concluded that the exposure of having distress and musculoskeletal disorders was minor in an ergonomically designed kitchen as compared to ordinary kitchen due to good planning of nominal reach zones in the kitchenette area.

Charu (2014) conducted a study on two hundred respondents of age group- 35 to 55 years, selected purposively from two regions of Ludhiana city [9]. She used NIOSH Discomfort Survey and Rated Professed Effort to measure the physiological stress. A Kitchen Aid was designed based on the respondent's anthropometric dimensions and assessed on 20 respondents in the research laboratory. Results exposed that most of the respondents having joint ache, back ache and severe weakness while working (ending, and stretching while storing) in the kitchen in standing posture. In the surveillance of workplace, it was noticed that few respondents had average height of cooking; preparation center and basin were 85.30 centimeters, 85.73 centimeters and 85.85 centimeters respectively, which were not based on the anthropometric dimensions of the respondents. On the basis of outcomes, a Kitchenette Aid (Fig. 3) was designed and assessed. It was observed that there is compelling decrease in heart rate, energy outflow, and physiological cost of work.

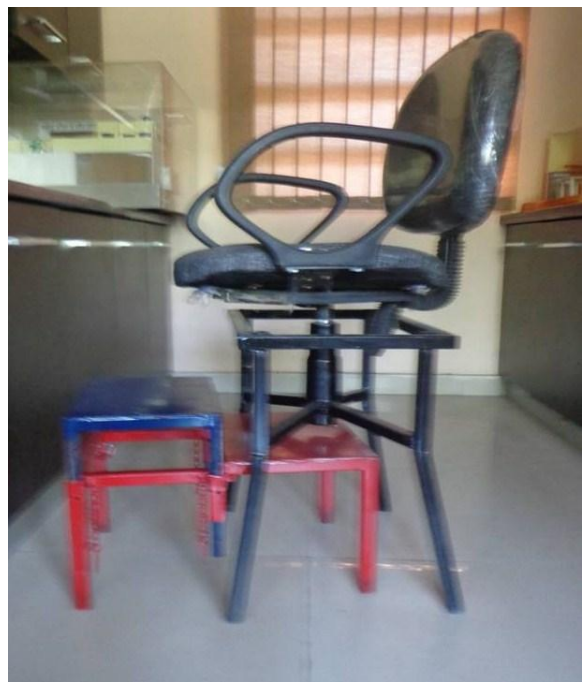

Fig. 3. The designed Kitchen Aid (Source: Developing Ergonomically Designed Kitchen Aid for Reducing Physiological Stress of Women Working in Standing Type Kitchen, 2014)

Mahajan, Swati Ashok and Patwardhan (2015) conducted a study based in which a data on two hundred sample of existing kitchenette were selected randomly [10]. The obtained data was used to design a kitchen which gives comfort while working. The study was executed using interview schedule and questionnaire methods. Anthropometric data was collected by means of measuring tape. The data collected were tabularized and analyzed. It was concluded in the study that while working in "L-Shaped" kitchen counters respondents were more comfortable.
Baroto Tavip Indrojarwo Eko Nurmianto, Ellya Zulaikha (1974) conducted a research which was dedicated for family of small habitat at Surabaya by qualitative and quantitative methodology [11]. The target community was selected for the reason that the existing conditions of their kitchenette were having several problems of comfort, safety, and health. The quantitative technique devised to classify worker characteristics, habitat and kitchenette kinds, cooking behavior and ergonomic aspect. The qualitative technique devised to classify recurrence table of cooking and family behavior. The techniques were created by considering speculations of kitchen configuration, zoning, structure and spread out. The study outcome was technologically advanced to a new conception of kitchen furniture design that minimized the kitchenette activities that need to be done by facing wall, so that during working, women can interact with others and do other jobs without difficulty. The kitchen design of the study result has module framework that can adaptably be balanced with any house structure.

Kiran Shete, Harshal Tukaram Pandve and Tanmayee Puntambekar (2015) conducted a study in which overall 104 patients took part [12]. Before the treatment, the Oswestry Disability Index (ODI) was taken. Then again ODI was taken after one week, after one month, six months and one year of the session. The questionnaire was intended to assemble information with respect to how the low back ache and the upper back ache had influenced the ability to deal with the day to day activities. They did changes in the workplace (kitchen) like taking hassock while standing; ordering cupboards and postural alterations were done. The objective of all these alterations was to decrease the superfluous loading of muscles, correction of the posture, and limit the weariness level. It was concluded in the study that the reduction in the disability index of 104 patients in a time span of one year was from an average of $35 \%$ (before one year) to $2 \%$ (following one year). And the research also concluded that these alterations decrease the lower and upper back ache, and discomfort and moreover it increased the functional capabilities.

\section{METHODS AND MATERIALS}

The research study was conducted on 30 participants from different cities of Punjab region. The participants were nominated for the investigation of average height $168.14 \mathrm{~cm} \pm$ 2.69 S.D. and normal age 36.4 years \pm 3.02 . Anthropometric data (Length of arm, length of elbow, full height, waist height) of each individual using measuring tape was collected. Two working environments of the kitchen, based upon the anthropometric data were prepared. The volunteers were provided with the questionnaire about, which kitchen environment they found most suitable and easy to work in One of the above prototypes was selected based upon the preferences and an idea of the parameters of various kitchen components was known.

Thereafter, the participants were asked to work in the standard-designed kitchen. The images of all the participants were captured in all postures while working in

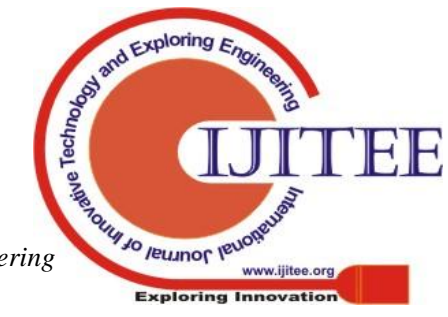


the kitchen. Mostly used postures will be taken into consideration. The awkward postures were analyzed using REBA and RULA Worksheet. The scores for various postures were filled in RULA and REBA worksheets (appendix).

The selected images of the women performing their normal activities in the kitchen are shown in Fig. 4. The observed data was critically analyzed. The final scores for REBA and RULA were computed. The ergonomic risk factors were recognized by analyzing the outcomes.

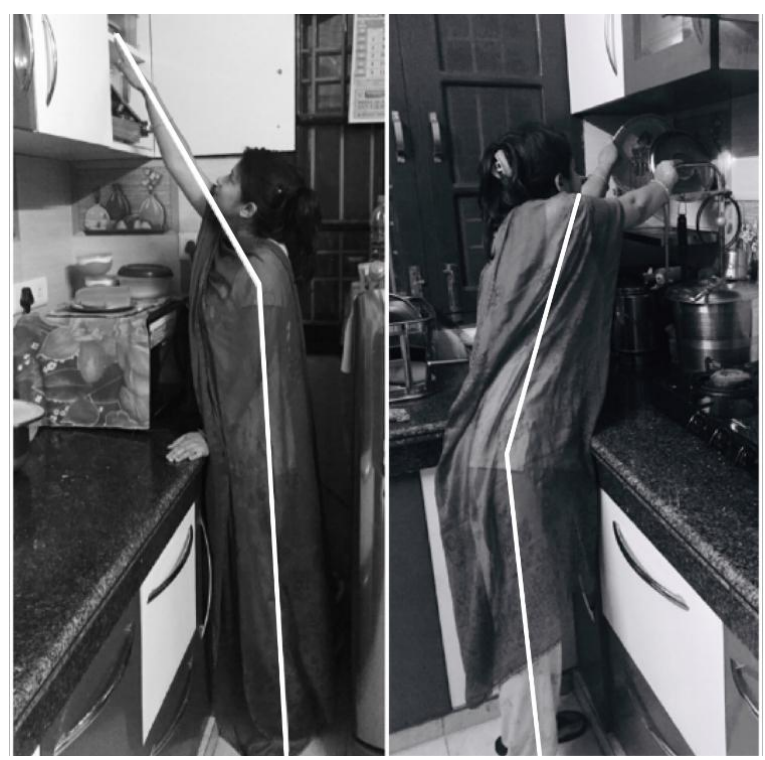

Fig.4.(a) Reaching

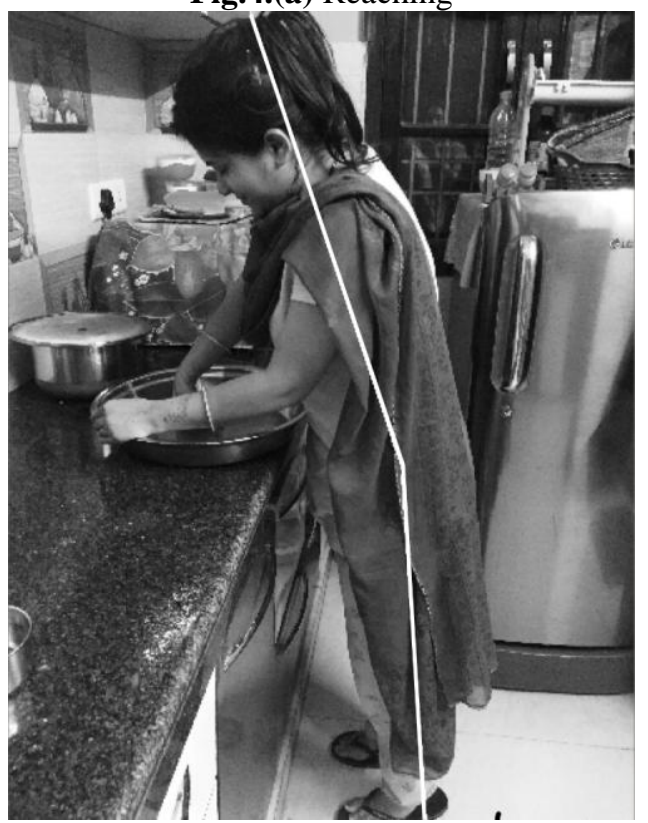

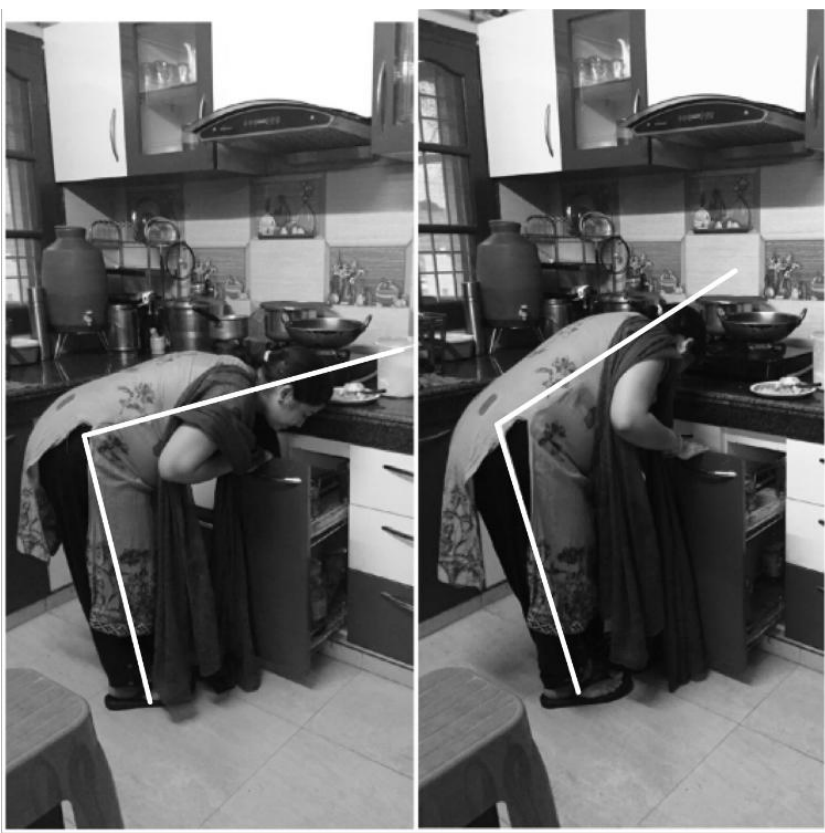

Fig.4.(b) Bending

Fig.4. Various postures adopted while working.

The volunteers were again asked to repeat the same procedure working in the kitchen designed on the basis of anthropometric data. The scores for various postures were again noted in the REBA and RULA Employee worksheets.

The REBA Employee Assessment Worksheet scores were classified into five ranges $1,2-3,4-7,8-10$ and $11+$, which indicates negligible risk, low risk (change may be needed), medium risk (further investigation, change soon), high risk (investigate and implement change) and very high risk (implement change) respectively. Similarly, the RULA Employee Assessment Worksheet scores were classified into four ranges 1-2, 3-4, 5-6 and 6+, which indicates negligible risk (no action required), low risk (change may be needed), medium risk (further investigation, change soon), and very high risk (implement change now) respectively.

\section{RESULTS AND DISCUSSION}

The REBA Employee Assessment Worksheet that was assessed is shown in the appendix. The level of risk of Musculoskeletal Disorders (MSD) based on the scores of REBA worksheet analysis while working in the kitchen is presented in the Table II. The RULA Employee Assessment Worksheet that was assessed is shown in the appendix. The level of risk of Musculoskeletal Disorders (MSD) based on the scores of RULA worksheet analysis while working is presented in the Table III.

Table II. Categorization under REBA level

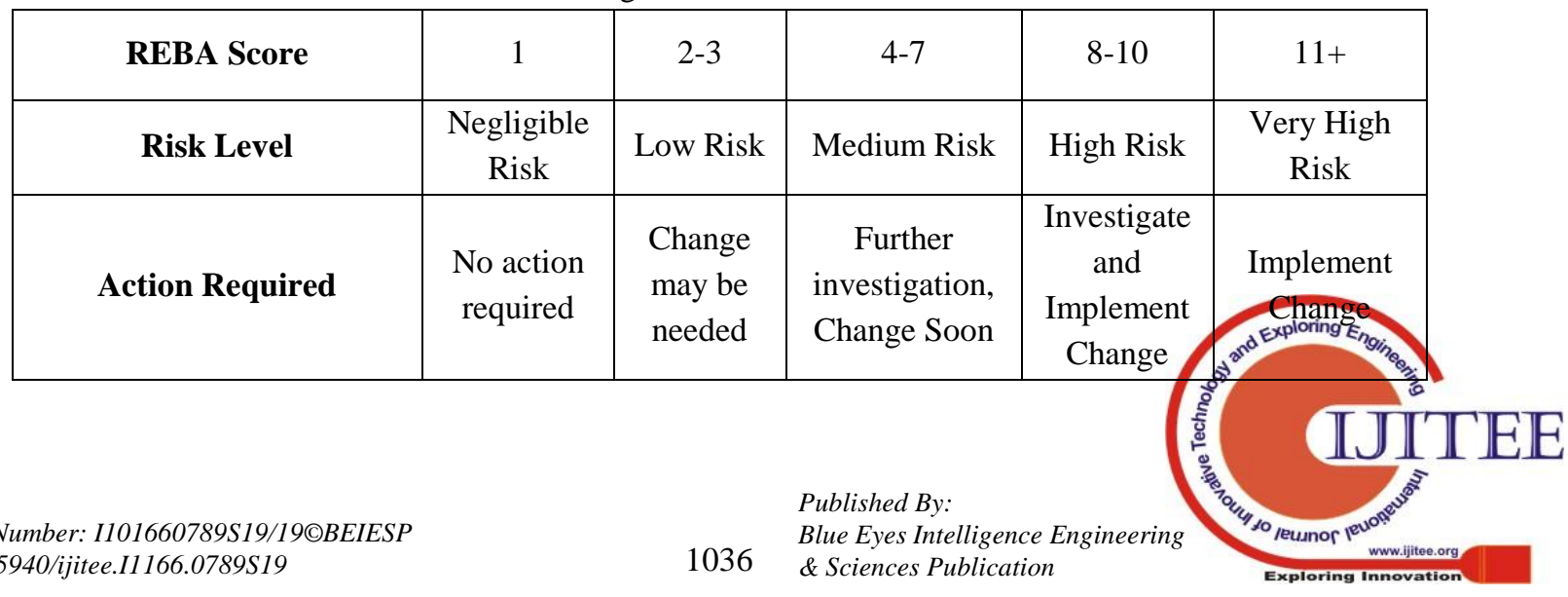




\begin{tabular}{|c|c|c|c|c|c|c|}
\hline \multirow{2}{*}{$\begin{array}{c}\text { Percentage } \\
\text { of Workers }\end{array}$} & $\begin{array}{c}\text { Standard } \\
\text { kitchen }\end{array}$ & -- & -- & 18 & 59 & 33 \\
\cline { 2 - 6 } & $\begin{array}{c}\text { Designed } \\
\text { kitchen }\end{array}$ & 47 & 42 & 11 & -- & -- \\
\hline
\end{tabular}

Table III. Categorization under RULA level

\begin{tabular}{|c|c|c|c|c|c|}
\hline \multicolumn{2}{|c|}{ RULA Score } & $1-2$ & $3-4$ & $5-6$ & $6+$ \\
\hline \multicolumn{2}{|c|}{ Risk Level } & $\begin{array}{c}\text { Negligible } \\
\text { Risk }\end{array}$ & Low Risk & Medium Risk & Very High Risk \\
\hline \multicolumn{2}{|c|}{ Action Required } & No action & Change may & $\begin{array}{c}\text { Further } \\
\text { investigation, }\end{array}$ & Implement \\
\hline \multirow{2}{*}{$\begin{array}{l}\text { Percentage } \\
\text { of Workers }\end{array}$} & $\begin{array}{c}\text { Standard } \\
\text { kitchen }\end{array}$ & -- & 4 & 47 & 49 \\
\hline & $\begin{array}{c}\text { Designed } \\
\text { kitchen }\end{array}$ & 53 & 38 & 9 & -- \\
\hline
\end{tabular}

Table 2 shows that while using standard designed kitchen, $33 \%$ of the women were at very high risk and the implementation of the change is required, whereas $59 \%$ of the women are at high risk and need to investigate and then change is to be implemented and $18 \%$ of the women require further investigation and change is needed soon. Whereas in the case of kitchen designed on the basis of anthropometry, the level of MSD risk is negligible in $47 \%$ of the women that means that no action is required, and $42 \%$ of the women are at low risk and $11 \%$ at medium risk.

Table 3 shows that while using standard designed kitchen, $49 \%$ of the women were at very high risk and the implementation of the change is required, whereas $47 \%$ of the women are at medium risk and needs further investigate and then change is to be implemented soon and $4 \%$ of the women are at low risk. Whereas in the case of kitchen designed on the basis of anthropometry, the level of MSD risk is negligible in $53 \%$ of the women that means that no action is required, and $38 \%$ of the women are at low risk and $9 \%$ at medium risk and require further investigation and change is needed soon.
Several women while working in the kitchen were bending their back beyond safe limit while bending to take utensils from lower shelves and maximum of them were having high strain in their upper arm. Women were recommended to preserve their back straight while working in the kitchen. Furthermore, during some other type of works in the kitchen the women were bending their back to a greater degree which was unacceptable and they required an urgent change.

\section{CONCLUSION}

It was analyzed by the results of the REBA Employee Assessment Worksheet that while working in the ergonomically kitchen the risk for musculoskeletal disorder is negligible in most of the cases than working in the standard-designed kitchen. On the basis of which it is concluded that there is need to design the kitchen ergonomically so as to reduce the work related musculoskeletal disorders among women working in the kitchen. In future, the work can be done to rationalize the kitchen dimensions that should be used in its designing. 


\section{APPENDIX}

ERGONEMICSS RULA Employee Assessment Worksheet Task Name: Date:

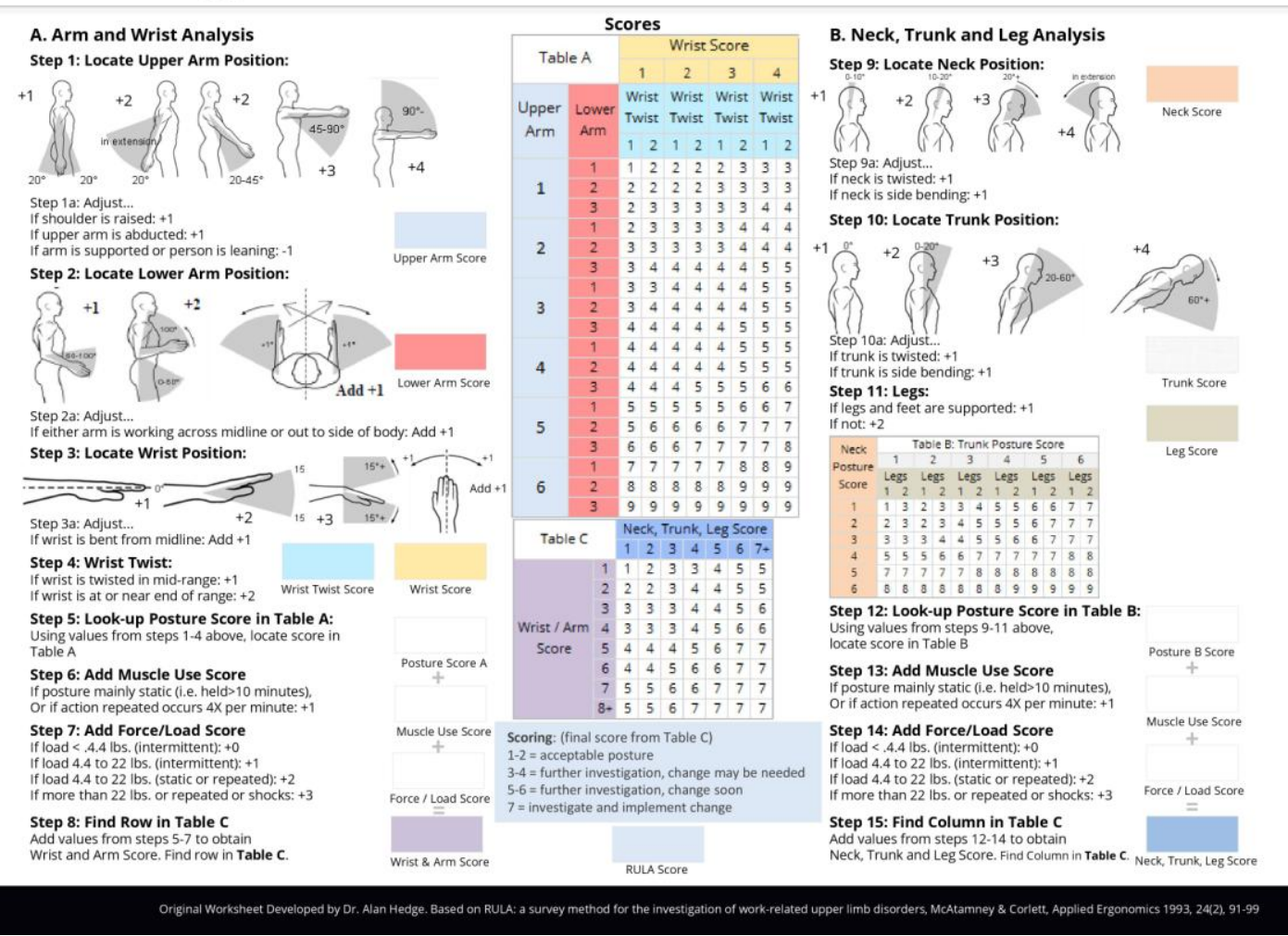

Source: https://ergo-plus.com/wp-content/uploads/RULA.pdf

\section{ERGONEMICS}

REBA Employee Assessment Worksheet

Task Name:

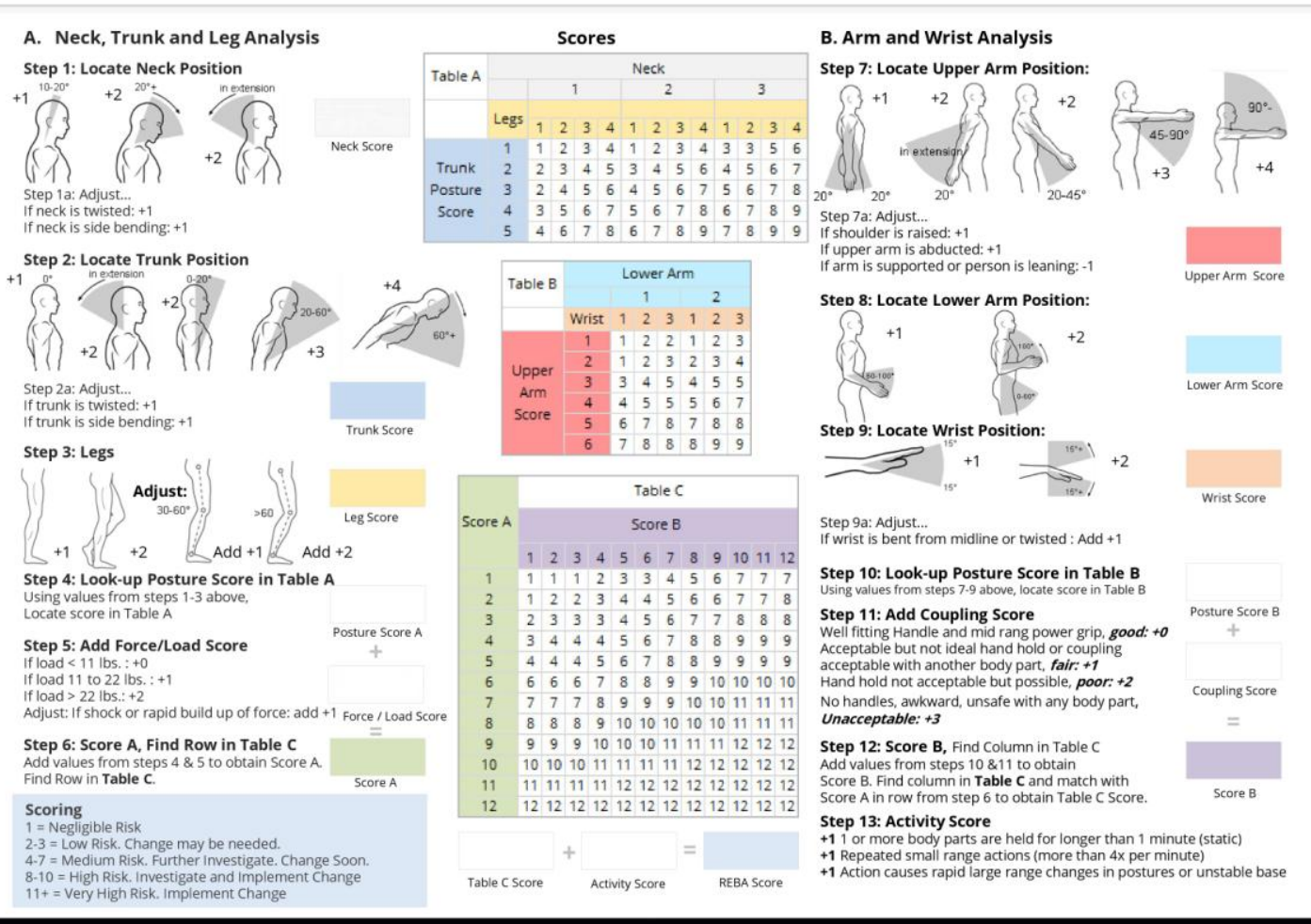

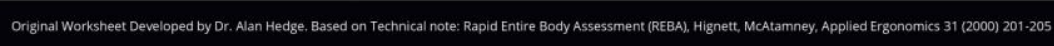

Source: https://ergo-plus.com/wp-content/uploads/REBA.pdf

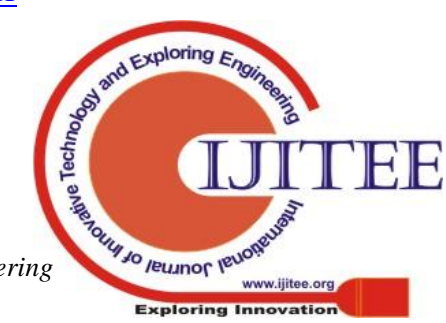




\section{REFERENCES}

1. J Kishtwaria, P Mathur and A Rana, "Ergonomic Evaluation of Kitchen work with reference to space designing", in J Hum Ecol, 2007 21:43-46.

2. Nowakowski, Przemyslaw, "Kitchen Chores Ergonomics: Research and Its Application", 2018, pp. 43-52.

3. Braton, N.J., "Occupational causes of disorders in the upper limb", in British Medical. J., 1992, pp. 309-311.

4. Sultana, Sajida and Prakash, Chitra, "The ergonomic perspective of the home makers in using kitchens", in Asian J. Home Sci., 2014, 9 (1): 25-28.

5.S Gangopadhyay, T Bandyopadhyay, "Ergonomics study on analysis of normal activities of Indian women in kitchen", in Biomedicine, 1999, pp. 123-128.

6. N Agrawal, D \& A Madankar, T \& S Jibhakate, M., "Study and Validation of Body Postures Of Workers Working In Small Scale Industry through RULA," in International Journal of Engineering Science and Technology, Vol. 3 No.10 October 2011

7. Maguire et al, 2011, "Age friendly kitchens: a study based on social history and ergonomics", in 6th International Conference on Inclusive Design: The Role of Inclusive Design in Making Social Innovation Happen. Royal College of Art, London, UK, 2011.

8. Lynn McAtamney and E Nigel Corlett, "RULA: a survey method for the investigation of world-related upper limb disorders", Applied Ergonomics, 1993 24(2):91-99.

9. Charu,. "Developing Ergonomically Designed Kitchen Aid for Reducing Physiological Stress of Women Working in Standing Type Kitchen”, 2014. Available:

http://krishikosh.egranth.ac.in/handle/1/5810016061

10. Mahajan, Swati Ashok and Patwardhan, S.L., "A study of ergonomic approach to kitchen work centers", Asian J. Home Sci., 2015, 10 (2) 371-374.

11. Baroto Tavip Indrojarwo Eko Nurmianto, Ellya Zulaikha, "Design Study of Ergonomic Kitchen for Small Dwelling With Family Interaction Concept".

12. Kiran Shete, Harshal Tukaram Pandve and Tanmayee Puntambekar. "Role of Ergonomics in Kitchen Related Back Problems", J Ergonomics 2015

\section{AUTHORS PROFILE}

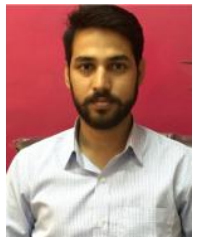

Amit Bhatia received his B.Tech. degree in Civil Engineering in 2014 from Punjab Technical University, Jalandhar, M.E. from PEC University, Chandigarh in 2016. He is Ph.D. Research Scholar In the Civil Engineering Department at RIMT University, Punjab. Presently, he is working as Managing Director, Gyanm College of Competitions, Una, H.P., India. He has published 2 papers in International Journals.

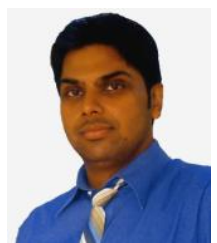

Dr Sandeep Singla received his B.Tech. degree in civil engineering in 2001 from Punjab Technical University, Jalandhar M.Tech from Thapar Institute of Engineering \& Technology, Patiala in 2004 \& PhD degree from National Institute of Technology (NIT) Kurukshetra in 2018. Presently, he is working as Professor \& Head in Department of Civil Engineering, RIMT University, Punjab, India. He has published more than 60 papers in National and International Journals/Conferences. He has guided more than 30 M.Tech thesis. Besides being member of board of studies in various universities, he is a life member of ISTE and also member of IEI. His research interests include environmental engineering, waste management, concrete technology, artificial intelligence, remote sensing and GIS. 